\title{
Experimental Mode Content Analysis Technique for Complex Overmoded Waveguide Systems
}

\author{
Ahmet G. Cepni*, Daniel D. Stancil, and Dagfin Brodtkorb ${ }^{\dagger}$, \\ Department of Electrical and Computer Engineering, \\ Carnegie Mellon University, Pittsburgh, PA 15213-3890, USA \\ Email: \{acepni,stancil\}@andrew.cmu.edu \\ † ABB Corporate Research, Bergerveien 12, P.O. Box 90, N-1735 \\ Billingstad, NORWAY dagfin.brodtkorb@no.abb.com
}

\begin{abstract}
In this paper, short time Fourier transform techniques are used to analyze the mode content of overmoded waveguides. The analysis is applied to transmission through straight sections, bends, and T-junctions. In addition to determining the modes with the dominant contributions to the response, the presence of mode conversion in bends and T-junctions is observed. The direct application of this technique is channel characterization of an HVAC duct system, which behaves as a multimode waveguide network at RF frequencies when used for indoor wireless communications.
\end{abstract}

\section{INTRODUCTION}

Use of heating, ventilation and air conditioning (HVAC) ducts for indoor communications is a topic of recent interest [1]. Ducts can be used to provide indoor wireless LAN coverage (IEEE $802.11 \mathrm{a} / \mathrm{b} / \mathrm{g}$ ). A typical HVAC duct system is a complex network of hollow metal pipes of rectangular or circular cross section which may contain multiple bends, T-junctions, tapers, etc. These ducts behave as multimode waveguides when driven at $\mathrm{RF}$ and microwave frequencies. The RF signal is coupled into ducts using antennas mounted on the duct walls. The antenna excites waveguide modes in the duct which propagate with different group velocities and attenuation constants. These modes are reflected and scattered into one another by terminations, nonuniformities and discontinuities before arriving at the receive antenna. The number of modes varies depending on geometry and dimensions of the HVAC duct system elements as well as the operating frequency. Multimodal dispersion and reflections from non-uniformities can significantly affect the signal quality, and thus the data transmission capacity of the HVAC network. Consequently, it is valuable to have a simple method for examining mode content at various locations in the HVAC duct system. Having such a method can assist in developing channel models as well as improved duct components and antennas. The method should be applicable to arbitrary systems containing components such as bends, T-junctions, and Y-junctions.

This paper presents a technique based on the short time fourier transform (STFT) that allows one to quickly analyze the mode content in complex HVAC duct systems. It requires a wideband channel measurement between the points of interest on the HVAC duct system. In contrast to the impulse response obtained by taking the inverse Fourier transform of the broadband frequency response, the STFT technique makes it possible to identify the presence of mode conversion as well as the relative contributions from each mode. The STFT is used to window the wave propagation in the waveguide, identifying the contribution of each mode to the received signal at discrete intervals of time. Mode content analysis using the STFT was done for straight waveguides in [2]. Using timefrequency analysis, the modal information, and scattering behavior in straight waveguides were examined. In this paper, we discuss the mode content analysis based on STFT technique for complex HVAC duct elements. 
There are several techniques that enable one to analyze the mode content in a waveguide. A brief summary of some of the available techniques is given in [3] along with a simple method for estimating the mode content in a straight duct from the frequency response. The remainder of this paper is organized as follows: The analysis of timefrequency behavior of waveguide modes is given in Section II, experiments are described in Section III, results and discussions are presented in Section IV, while Section V concludes the paper.

\section{AnAlysis}

In a waveguide environment, the electric field at a distance of $\mathrm{L}$ from the transmitter can be given as:

$$
E(x, y, z, f)=\sum_{n=1}^{N} C_{n} \vec{e}_{n} e^{ \pm \gamma_{n} z} e^{j 2 \pi f t},
$$

where $\vec{e}_{n}$ is the normalized electric field of the mode n, $C_{n}$ is the mode excitation coefficient, $\gamma_{n}=\alpha_{n}+j \beta_{n}$ is the complex propagation constant, $\alpha_{n}$ is the attenuation constant and $\beta_{n}$ is the propagation constant. In a complex HVAC duct channel, reflections from terminations, nonuniformities along the propagation path cause scattering in the E-field expression. [4] analytically discusses the channel transfer matrix of any arbitrary complex HVAC duct system that includes bends, T-junctions and metal terminations.

In a general waveguide environment, energy is carried in multiple propagating modes. The phase constant of a mode is given by:

$$
\beta=\frac{2 \pi f}{c} \sqrt{1-\left(\frac{f_{c}}{f}\right)^{2}},
$$

where $f$ is the frequency, $f_{c}$ is the cutoff frequency of the mode, and $\mathrm{c}$ is the velocity of light in free space. The group velocity for a propagating mode in a waveguide is given by:

$$
u_{g}=\frac{2 \pi}{d \beta / d f}=c \sqrt{1-\left(\frac{f_{c}}{f}\right)^{2}}
$$

The mode pack travels a distance of $\mathrm{L}$ in $t=L / u_{g}$ seconds, or

$$
t=\frac{L}{u_{g}}=\frac{L}{c \sqrt{1-\left(\frac{f c}{f}\right)^{2}}},
$$

This equation indicates that at a particular frequency, the mode which has the lowest cut-off frequency arrives to the receive antenna in the shortest time span. As the operating frequency gets closer to the mode cut-off frequency, the group velocity becomes smaller and the mode pack moves slowly. This equation defines the time-frequency behavior of each mode in a waveguide and can also be written as:

$$
f=\frac{t f_{c}}{\sqrt{t^{2}-\left(\frac{L}{c}\right)^{2}}} .
$$




\section{EXPERIMENTS}

To characterize the mode content behavior of HVAC ducts, we made measurements using straight ducts, a bend, and a T-junction, as shown in Fig. 1. The frequency responses over the range $0.5-3 \mathrm{GHz}$ were measured between the points indicated using an Agilent E8358A Vector Network Analyzer. We used cylindrical ducts $0.3 \mathrm{~m}$ in diameter made of galvanized steel. The signal was coupled to the duct using monopole antennas of $3.1 \mathrm{~cm}$ length (approximately quarter wavelength at $2.45 \mathrm{GHz}$ ) inserted into the duct wall. In all of the measurements described, the ends of the duct networks were open, approximating matched loads.

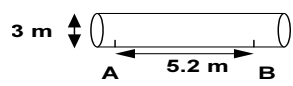

(a)

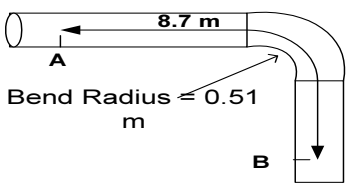

(b)

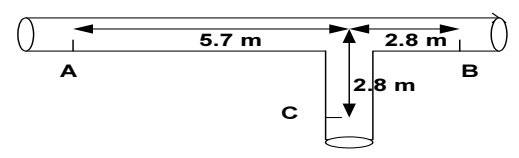

(c)

Fig. 1. Experimental testbed. (a) Straight duct (b) duct with a bend (c) duct with a T-junction.

\section{Results}

In the analysis of waveguide modes in HVAC ducts, wideband frequency data are used to generate an impulse response via an inverse Fourier transform (IFT). The frequency response and impulse responses for all cases are shown in Fig. 2. Measurements were made using 1601 points. The wideband channel measurement $(0.5-3 \mathrm{GHz})$ resulted in a time resolution of $0.4 \mathrm{~ns}$. The resulting time span was $640 \mathrm{~ns}$. For the straight duct, the average channel gain was $-20.2 \mathrm{~dB}$, for the bend it was $-24.6 \mathrm{~dB}$. For the T-junction it was $-28.4 \mathrm{~dB}$ (path A to B) and $-31.3 \mathrm{~dB}$ (path A to $\mathrm{C}$ ). The measured impulse responses were used to obtain STFT responses, yielding time-frequency phase-space representations of the data. STFT calculates the spectrogram of the impulse response in 2-D space (time vs frequency). First, the time domain signal is splitted into overlapping segments. Each segment is fourier transformed (512-point FFT) using a Hanning window in time of length 64. The time-frequency analysis (see Fig. 3) shows that the energy is predominantly in five modes. The calculated f-t curves from Eqn. 5 for each mode successfully predicts the time-frequency behavior of each mode. The modes have cutoff frequencies at 0.9568 , $1.3162,1.6661,2.01012 .3500 \mathrm{GHz}$ corresponding to the $T E_{21}, T E_{31}, T E_{41}, T E_{51}$, and $T E_{61}$ modes. In the case of straight ducts (see Fig. 3), the most excited mode is $T E_{51}$ and it has $45 \%$ of the total energy. The second most excited mode is $T E_{41}$ and it has $29 \%$ of the total energy. As for the bend, the most excited mode is again $T E_{51}$ and it has $46 \%$ of the total received energy. The direct path of the T-junction has $T E_{41}$ as the strongest mode $(35 \%)$. The vertical path has $T E_{51}$ as the mode with the most amount of energy (45\%).

Use of the STFT method enables the dominant mode content to be easily identified. Further, the presence of mode conversion through various elements is indicated by changes in the relative mode content relative to the straight duct, and the presence of increased energy between the mode curves in the time-frequency diagrams.

\section{Conclusions}

The mode behavior in complex HVAC duct elements was discussed in this paper. The experiments showed that using STFT technique, it is possible to estimate the dominant modes in the HVAC duct system and energy distribution among the modes. This technique is simple to implement and valid for complex waveguide systems that might include 


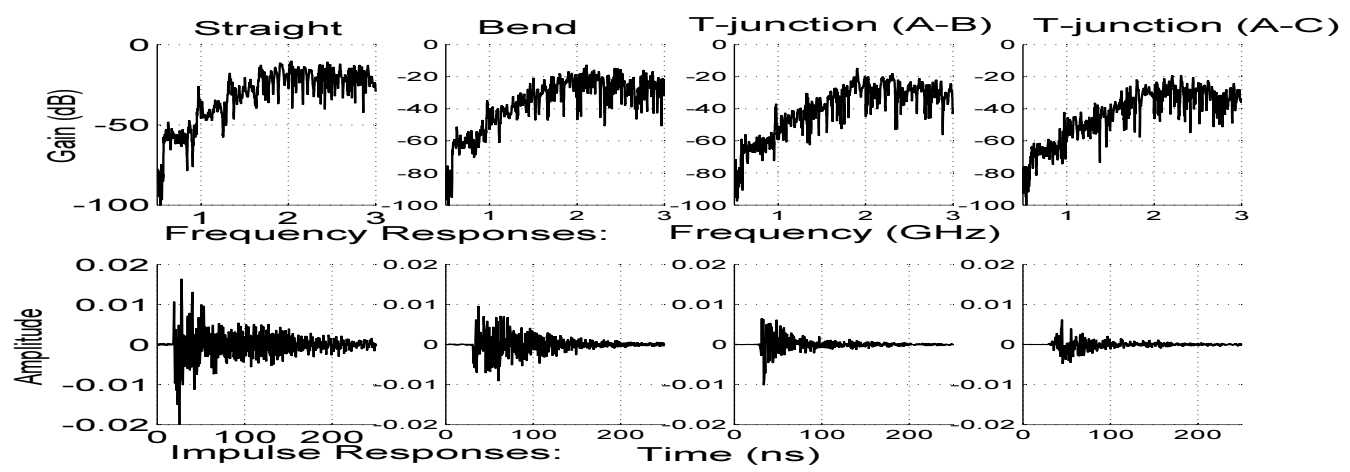

Fig. 2. The frequency response measurements and the resulting impulse responses.
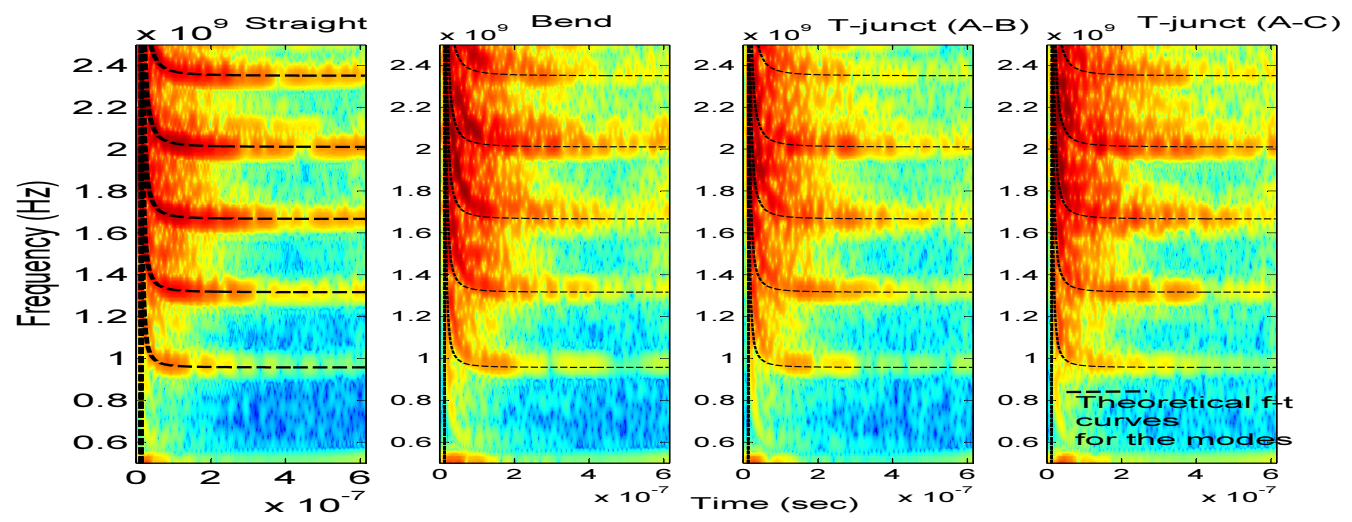

Fig. 3. Time-frequency diagrams with dispersion curves from Eqn. 5 shown as dashed lines.

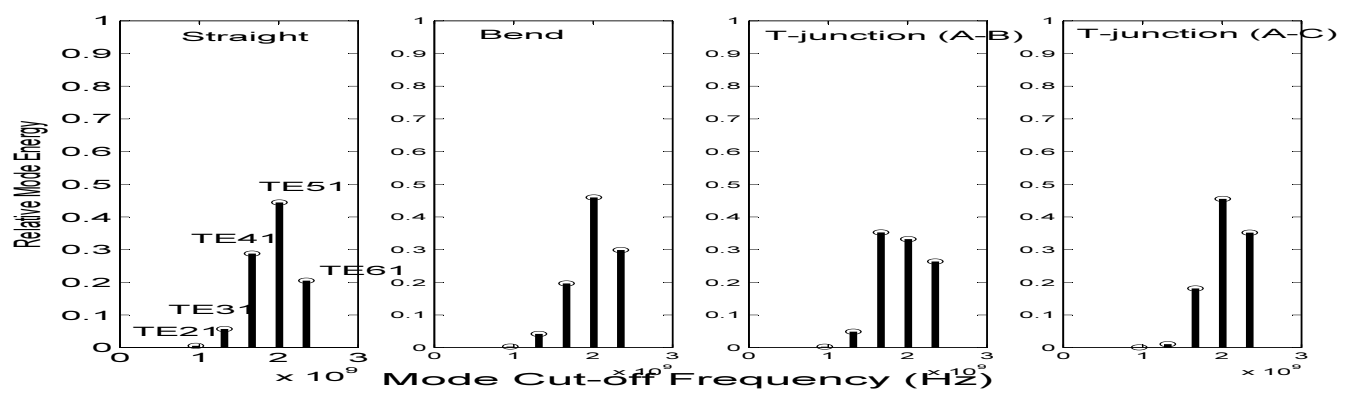

Fig. 4. The relative energy in the observed modes. The modes are ordered by cutoff frequencies (horizontal axis).

bends, T- and Y- junctions.

\section{REFERENCES}

[1] D. D. Stancil, O. K. Tonguz, A. Xhafa, A. Cepni, P. Nikitin, and D. Brodtkorb, "High speed internet access via HVAC ducts: A new approach," in Proc. of IEEE Global Telecomm. Conf. (GLOBECOM'01), vol. 6, pp. 3604-3607, San Antonio, Texas, Nov. 2001.

[2] A. Moghaddar, E. Walton, "Time-frequency distribution analysis of waveguide modes ," Antennas and Propagation Society International Symposium, 1992. AP-S. 1992 IEEE , 18-25 July 1992 Pages:2114 - 2117 vol.4

[3] P.V. Nikitin, D.D. Stancil, A.G. Cepni, A.E. Xhafa, O.K. Tonguz, D. Brodtkorb, "A novel mode content analysis technique for antennas in multimode waveguides," Microwave Theory and Techniques, IEEE Transactions on ,Volume: 51 , Issue: 12 , Dec. 2003 Pages:2402 - 2408

[4] P.V. Nikitin, D.D. Stancil, A.G. Cepni, A.E. Xhafa, O.K. Tonguz, D. Brodtkorb, "Propagation modelling of complex HVAC networks using transfer matrix method," Antennas and Propagation Society International Symposium, 2003. IEEE ,Volume: 2, June 22-27, 2003 Pages:126 - 129. 